\title{
Algorithm Science to Operations for the National Polar-orbiting Operational Environmental Satellite System (NPOESS) Visible/Infrared Imager/Radiometer Suite (VIIRS)
}

\author{
James L. Duda \\ NPOESS Integrated Office \\ NASA/GSFC Code 402 \\ Silver Spring, MD 20910 \\ James.L.Duda@nasa.gov
}

\author{
Suzanna C. Barth \\ Raytheon Company, NPOESS \\ 16800 E. Centretech Parkway \\ Aurora, CO 80011 \\ scbarth@raytheon.com
}

\begin{abstract}
The VIIRS sensor provides measurements for 22 Environmental Data Records (EDRs) addressing the atmosphere, ocean surface temperature, ocean color, land parameters, aerosols, imaging for clouds and ice, and more. That is, the VIIRS collects visible and infrared radiometric data of the Earth's atmosphere, ocean, and land surfaces. Data types include atmospheric, clouds, Earth radiation budget, land/water and sea surface temperature, ocean color, and low light imagery. This wide scope of measurements calls for the preparation of a multiplicity of Algorithm Theoretical Basis Documents (ATBDs), and, additionally, for intermediate products such as cloud mask, et al. Furthermore, the VIIRS interacts with three or more other sensors. This paper addresses selected and crucial elements of the process being used to convert and test an immense volume of a maturing and changing science code to the initial operational source code in preparation for launch of NPP. The integrity of the original science code is maintained and enhanced via baseline comparisons when re-hosted, in addition to multiple planned code performance reviews.
\end{abstract}

\section{INTRODUCTION AND BACKGROUND}

The National Polar-orbiting Operational Environmental Satellite System includes a constellation of three satellites, associated telemetry stations, and four ground processing elements. These physical facilities are supported by software and activities which enable the conversion in near real-time of raw telemetry data from spacecraft instruments to produce Sensor Data Records (SDRs) ${ }^{1}$, Environmental Data Records $(\mathrm{EDRs})^{2}$, as well as additional outputs including Intermediate Products and Quality Flags. The

\footnotetext{
${ }^{1}$ Sensor Data Records (SDRs) are full resolution sensor data that are time referenced, Earth located, and calibrated by applying the ancillary information, including radiometric and geometric calibration coefficients and geo-referencing parameters such as platform ephemeris.

${ }^{2}$ EDRs are geophysical parameters that range from atmospheric products detailing cloud coverage, temperature, humidity, and ozone distribution; to land surface products showing snow cover, vegetation, and land use; to ocean products depicting sea surface temperatures, sea ice, and wave height; to characterizations of the space environment.
}

VIIRS sensor is manifested on all three of these orbits. This paper discusses selected aspects of the process of converting science algorithms to operational use for a single sensor, VIIRS. The VIIRS operational algorithms are capable of calculating and delivering environmental data records in near real-time. The process is applicable to both NPOESS and the NPOESS Preparatory Project (NPP).

The algorithms used to create these data records are delivered as science grade software developed in conjunction with the spacecraft instrument. The algorithm's theoretical foundation is described in associated Algorithm Theoretical Basis Documents (ATBDs). Test data are delivered by the algorithm subcontractor to the prime, Northrop Grumman Space Technology (NGST). NGST then re-hosts the code and verifies the test data and results provided by the algorithm vendor, recording performance metrics of the algorithm and identifying potential follow-on work. NGST also prepares a draft version of an Operational Algorithm Document (OAD) specifying additional Quality Flags and Threshold Tables for the algorithm ${ }^{3}$. This package, including the algorithm code, the ATBD, draft Operational Algorithm Document (OAD), test data, and any additional algorithm subcontractor documentation is passed to Raytheon for conversion to operational code. The transfer of these algorithms and data is performed via a multi-stage, boarded review process involving the subcontractor algorithm lead, NGST algorithm lead, Raytheon operational algorithm lead, and general science and architecture lead. Members of the science community and the Integrated Program Office are also invited.

The conversion process for all of the NPOESS sensors' algorithms to operational code follows five steps, including (1) Porting the algorithm code to an IBM AIX (Unix)

\footnotetext{
${ }^{3}$ The OAD is a description of the computer science implementation of the algorithm, using software architecture, pseudo code and/or flowcharts.
} 


\section{Report Documentation Page}

Form Approved

OMB No. 0704-0188

Public reporting burden for the collection of information is estimated to average 1 hour per response, including the time for reviewing instructions, searching existing data sources, gathering and maintaining the data needed, and completing and reviewing the collection of information. Send comments regarding this burden estimate or any other aspect of this collection of information,

including suggestions for reducing this burden, to Washington Headquarters Services, Directorate for Information Operations and Reports, 1215 Jefferson Davis Highway, Suite 1204, Arlington

VA 22202-4302. Respondents should be aware that notwithstanding any other provision of law, no person shall be subject to a penalty for failing to comply with a collection of information if it

does not display a currently valid OMB control number.

\begin{tabular}{|c|c|c|}
\hline $\begin{array}{l}\text { 1. REPORT DATE } \\
\mathbf{2 5} \text { JUL } \mathbf{2 0 0 5}\end{array}$ & $\begin{array}{l}\text { 2. REPORT TYPE } \\
\text { N/A }\end{array}$ & $\begin{array}{l}\text { 3. DATES COVERED } \\
\text { - }\end{array}$ \\
\hline \multirow{3}{*}{\multicolumn{2}{|c|}{$\begin{array}{l}\text { 4. TITLE AND SUBTITLE } \\
\text { Algorithm Science to Operations for the National Polar-orbiting } \\
\text { Operational Environmental Satellite System (NPOESS) Visible/Infrared } \\
\text { Imager/Radiometer Suite (VIIRS) }\end{array}$}} & 5a. CONTRACT NUMBER \\
\hline & & 5b. GRANT NUMBER \\
\hline & & 5c. PROGRAM ELEMENT NUMBER \\
\hline \multirow{3}{*}{\multicolumn{2}{|c|}{ 6. AUTHOR(S) }} & 5d. PROJECT NUMBER \\
\hline & & 5e. TASK NUMBER \\
\hline & & 5f. WORK UNIT NUMBER \\
\hline \multicolumn{2}{|c|}{$\begin{array}{l}\text { 7. PERFORMING ORGANIZATION NAME(S) AND ADDRESS(ES) } \\
\text { NPOESS Integrated Office NASA/GSFC Code } 402 \text { Silver Spring, MD } \\
\mathbf{2 0 9 1 0}\end{array}$} & $\begin{array}{l}\text { 8. PERFORMING ORGANIZATION } \\
\text { REPORT NUMBER }\end{array}$ \\
\hline \multirow{2}{*}{\multicolumn{2}{|c|}{ 9. SPONSORING/MONITORING AGENCY NAME(S) AND ADDRESS(ES) }} & 10. SPONSOR/MONITOR'S ACRONYM(S) \\
\hline & & $\begin{array}{l}\text { 11. SPONSOR/MONITOR'S REPORT } \\
\text { NUMBER(S) }\end{array}$ \\
\hline
\end{tabular}

12. DISTRIBUTION/AVAILABILITY STATEMENT

Approved for public release, distribution unlimited

13. SUPPLEMENTARY NOTES

See also ADM001850, 2005 IEEE International Geoscience and Remote Sensing Symposium Proceedings (25th) (IGARSS 2005) Held in Seoul, Korea on 25-29 July 2005.

14. ABSTRACT

15. SUBJECT TERMS

16. SECURITY CLASSIFICATION OF:

a. REPORT

unclassified b. ABSTRACT

unclassified c. THIS PAGE

unclassified
17. LIMITATION OF ABSTRACT

UU
18. NUMBER

OF PAGES

4 19a. NAME OF

RESPONSIBLE PERSON 
platform $^{4},(2)$ Design and implementation of distinguishable Input, Processing, and Output processes, making use of standard I/O library functions, (3) Implementation of error handling and data quality reporting routines, (4) Optimization for algorithm execution speed, in order to meet designated latency targets, and (5) Implementation of fallback processing in case inputs are missing. A single algorithm generally depends on several inputs, including fields from National Center for Environmental Prediction (NCEP), as well as output fields from other associated algorithms. The final operational version of the algorithm generally relies on the integration of the algorithm with an algorithm chain such that the final operational version of the algorithm may be fully tested, delivered, and validated.

The VIIRS sensor is a follow-on instrument to the United States' National Aeronautical and Space Administration's Moderate Resolution Imaging Spectroradiometer (MODIS) which uses 36 spectral bands ranging from $620 \mathrm{~nm}$ to $14.385 \mu \mathrm{m}$ [2], while VIIRS uses 21 bands ranging from $412 \mathrm{~nm}$ to $11450 \mathrm{~nm}$. The instruments share some similarities, such as rotating double-sided mirror, solar diffuser and solar diffuser stability monitor, which create similarities between the MODIS and VIIRS Sensor Data Record (SDR) processing code, for calibration and geolocation.

\section{OVERVIEW OF VIIRS SCIENCE TO OPERATIONAL ALGORITHM CONVERSION}

The Visible/Infrared Imager/Radiometer Suite (VIIRS) takes measurements within the visible and infrared portion of the electromagnetic spectrum. The VIIRS instrument measures reflectance's, brightness temperatures, and equivalent black body temperatures in order to derive atmospheric, land and water surface parameters producing twenty-two EDRs. Of these 22 EDRs, the VIIRS is the primary instrument for eleven EDRs and secondary or contributory to eleven others. In the context of conversion from science to operations a certain number of the EDRs pose less of a challenge to convert. And, conversely, a number pose greater challenges to convert from science grade code to operational code.

Factors contributing to the conversion complexity include inter sensor mapping, cross granule processing, geolocation, ancillary data, intermediate products, algorithm maturity, and science complexity. Conversion simplicity for more straight-forward EDRs is characterized by fewer interdependencies and data independence from other sensors. Imagery EDRs using the visible light channels fit this category.

More complex EDRs include sea surface temperature, cloud base height, and aerosol optical properties. More complex EDRs may require multiple steps like cloud

\footnotetext{
${ }^{4}$ The AIX (Unix) platform has been selected by the NPOESS program as the hosting computer for the Integrated Data Processing System portion of the Ground Segment.
}

clearing, large matrix computations, searching Look Up Tables (LUTs) generated by radiative transfer models, etc.

\section{A. Development of VIIRS Science Grade Algorithm Code}

The VIIRS Science algorithms were developed under an independent subcontract to the government by Raytheon ITSS. These science algorithms included SDR code for sensor Calibration and Geolocation as well as the EDR code for each of the deliverable EDR products. Test data for the science code came from MODIS (also called proxy test data) and from NGST's Integrated Weather Test Bed Facility (simulated data). Further development after the science code was delivered to NGST included integration of Quality Flags (also including processing flags, identifying different algorithm branching performed).

Because imagery EDRs are created directly from the VIIRS Imagery band SDRs, NGST delivered the SDR code with technical instructions describing which map projection to use, adjustments for sensor visual effects such as "bowtie," and EDR product contents. The Day Night Band on VIIRS is also treated as imagery SDR, and is used to create the Near Constant Contrast EDR; however, a science version of this algorithm was delivered for conversion to operational code.

Some of the more complex EDR algorithms such as Cloud Base Height perform physical retrievals ${ }^{5}$, where the cloud effective particle size and cloud optical thickness are calculated, temperature profiles are used to estimate lapse rates, and parallax corrections made, in order to calculate the Cloud Base Height EDR.

\section{B. Conversion Of Science Grade Algorithm Code To Operational Code}

The five step conversion process from science to operational code is characterized by two Detailed Design Peer Reviews (DDPRs), first at the beginning of the conversion process to establish common Input-ProcessingOutput architecture, as well as Error Handling and Data Quality Reporting, and a second DDPR is held mid-way through the conversion for the design of Graceful Degradation (fallback processing) and Optimization (latency targeting). These two DDPRs are held for each SDR and EDR algorithm, and science algorithm leads from the sensor vendor, NGST, and the science community is invited to participate in addition to the operational algorithm lead and ground segment science leads. After each of the DDPRs is held and design has been approved and implemented, a Code and Unit Test (CUT) Review is held to vet the test results, confirming that the conversion is not deviating from the science results. There is one CUT for each of the DDPRs.

\footnotetext{
${ }^{5} \mathrm{~A}$ physical retrieval is a method of deriving the value of an atmospheric variable by numerically solving the radiative transfer equation until calculated radiances match observed radiances within tolerance.
} 
At each stage of conversion, after the DDPRs and CUTs, the science unit test data delivered with the algorithm to Raytheon are run through the operational version of the code on the IBM AIX platform, and formal benchmark test results are returned to NGST for verification that the operational conversion of the algorithm is within prespecified accuracy, precision, and uncertainty.

Another task performed during the conversion process, is to populate the $\mathrm{OAD}$ with architecture information, direction from NGST regarding checking for Quality Flags and Threshold Tables, and to describe substitute ancillary data written into the operational version of the science code.

Follow-on tasks described by NGST in Technical Memos or delivered as additional science code are incorporated into the operational algorithm, and descriptions of these changes are added to the OAD. Issues arising out of algorithm chain testing and integration are accommodated through additional reviews and testing.

Finally, the conversion of the algorithms once they have been unit tested proceeds to chain testing and integration, as well as a more robust latency analysis. Two of the most interdependent algorithm categories include the cloud and aerosol algorithms.

As an example, the testing and integration of the Cloud algorithms includes two types of chain testing and integration: (1) The execution of the Cloud algorithm chain itself (in order, Cloud Effective Particle Size, Cloud Optical Thickness, Cloud Top Height, Cloud Top Temperature, Cloud Top Pressure, Cloud Cover and Layers, and Cloud Base Height), and (2) The dependency of some of the Land algorithms (Land Albedo, Land Surface Temperature, and Vegetative Index) on the Cloud algorithms (Cloud Optical Thickness, Cloud Effective Particle Size).

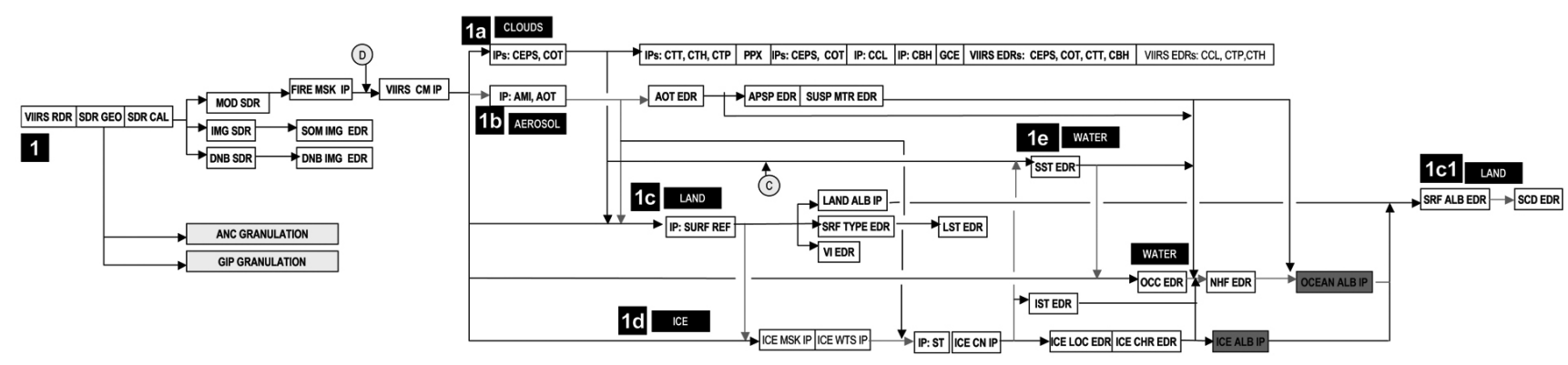

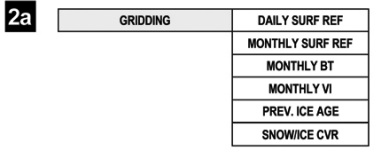

$2 b$

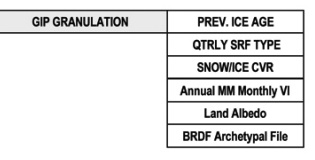

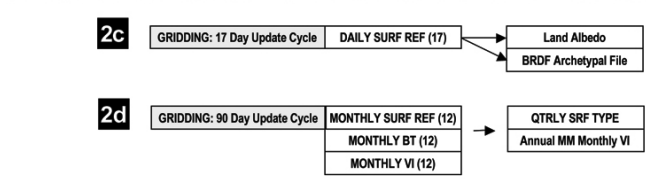

Sea lee Characteization EDR (Not in EDR IR V1.7)

Surface Type, Snow Cover/Jepth EDR \{ERR IR V1.7 adds Acivive Fires + Some Gracefu Degracation dependencies

Cloud Mask IP, Sea Surtace Temperature EDR (EDR IR V1.7 does not have input for SST)

Snow CoverDDepht EDR (in EDR IR V1.7)

Surtace Type EDR (in EDR IR v1.7)

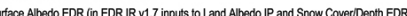

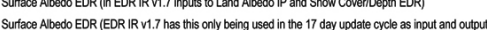

21

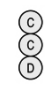

(6)

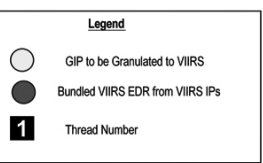

Figure 1. VIIRS Wiring Diagram describing the operating sequence of the VIIRS algorithms, Intermediate Products, and Raw, Sensor, and Engineering Data Records.

\section{ViIRs Sensor Challenges}

\section{A. Challenges in Verification of VIIRS Science Grade Code Performance}

Test Data of two types has been used for unit testing of individual algorithms: (1) Proxy data from heritage instruments and (2) simulated data from radiative transfer models. In the case where proxy data is available coincident with field mission data, there has been a preference to use the in situ data collected during field missions to verify proxy data from heritage instruments.
CAMEX field data was used for comparison purposes with the MODIS cloud algorithms ${ }^{6}$.

Chain testing of the algorithms will consist of two phases: (1) Functional testing making use of proxy test data and ensuring the NPOESS ground system includes the algorithm chain functionality; and (2) Performance testing to make use of model test data and to check the accuracy, precision, and uncertainty of each of the EDRs.

Both Cloud Optical Thickness and Aerosol Optical Thickness have a considerable number of downstream

\footnotetext{
${ }^{6}$ The Convection And Moisture EXperiment (CAMEX) is a series of field research investigations sponsored by the Earth Science Enterprise of the National Aeronautics and Space Administration (NASA).
} 
dependencies with Land, Ocean, and Surface Temperature algorithms. The sensitivity of these downstream algorithms to Optical Thickness values is a difficult attribute to capture, due to the wide variation of scene conditions covering the globe and the sequence of algorithm availability. Once the full chain of VIIRS algorithms has been integrated and tested with a global test data set, the sensitivity of the downstream algorithm performance will be measured.

\section{B. Challenges in Migration of Science Grade Code to Operational Code}

A challenge in development of algorithm performance has been the proliferations of Quality Flags, used to not only identify scene conditions affecting a measurement, but also sensor threshold states. Specifically, the spatial resolution of the VIIRS instrument causes Quality Flags calculated at the pixel level to balloon the storage requirements of the SDR and EDR products.

The Cloud and Aerosol algorithm have a significant number of downstream dependencies as well as being used to identify either degraded algorithm performance or conditions where the algorithm cannot correctly adapt to the physical conditions in the atmosphere, and at which point the performance is excluded from skill measurements. An example of a particular Quality Flag that translates into flagging such pixels is for Field of View cases where the Aerosol Optical Thickness exceeds 1.0. [4].

An example of where Quality Flags endanger product storage requirements of proliferating is in the case of the VIIRS Cloud Mask Intermediate Product. This product is comprised of four values actually describing the cloud state (cloudy, partially cloudy, clear, and partially clear), as well as twenty-eight Quality Flags per pixel identifying other scene conditions (cloud phase, presence of thin cirrus, fire, etc.). Due to the high spatial resolution, wide swath width, and high data volume of the VIIRS products, careful attention has been given to the management, design, and systems engineering of VIIRS Quality Flags.

\section{REWARDS AND HAZARDS}

Benefits of having an operational version of the VIIRS algorithms include performance optimization, modularization to facilitate later improvements, accommodating unavailability of expected input data, allowing for graceful degradation, and handling of recurring but rare orbital events.

\section{A. Rewards of VIIRS Science to Operations Conversion}

The above stated benefits are derived from the process of integrating the algorithms into a functioning, interdependent chain. This process includes accounting for sensor hardware behavior in the SDR algorithm, and taking into account the behavior of the sensor in the context of the spacecraft (thermal effects, orbital maneuvers, space environment).

Because the individual algorithms have generally been developed independently, a unified operational plan has resulted in formalized requirements and specifications for a systems architecture.

\section{B. Hazards of VIIRS Science to Operations Conversion}

The treatment of many of the science algorithms as stand alone calculations allows the independent verification of each algorithm's performance. However, the development and delivery to the Integrated Data Processing Segment of the EDR algorithms prior to completion and testing of the SDRs, has complicated the procedures used for Algorithm Chain Testing.

\section{SUMMARY}

This paper addressed selected elements of the process being used to convert and test the maturing and changing VIIRS science grade algorithm code to the initial operational algorithm source code. The paper described VIIRS EDRs that were particularly vexing and challenging to convert. Many reviews are performed during the conversion in order to maintain the integrity of the original science code.

It may benefit the scientists to become familiar with all aspects of this process, and, especially the modular form (I$\mathrm{P}-\mathrm{O})$ of the original science code. Running this code on their own platforms will facilitate their familiarity with the benefits and features of operational science code, diagnosis of on-orbit anomalies, code maintenance and future improvements. Using the operational version of the science code will also facilitate future algorithm improvement development and minimize the time for testing, verification, and validation, and configuration control for operational implementation of improved algorithms.

\section{REFERENCES}

[1] James L. Duda, Pamela G. Emch, "Science to Operations: Challenges, Rewards, and Pitfalls of Converting Algorithm Science Code to Operational Code", PROCEEDINGS OF SPIE Volume 5658, pp. 2027, November 10, 2004.

[2] MODIS Homepage, http://modis.gsfc.nasa.gov/, April 11, 2005

[3] NOAA NPOESS Homepage,

http://www.ipo.noaa.gov/About/sat evolu.html, April 12, 2005.

[4] NPOESS System Specification, Appendix D, October, 2004. 